\title{
Разработка нейроморфного акселератора
}

\author{
Д.Е. Ипатов ${ }^{1)}$, А.В. Зверев ${ }^{2)}$ \\ ${ }^{1}$ Институт физики полупроводников им. А.В. Ржанова СО РАН, Новосибирск, 630090, пр. Ак. \\ Лаврентьева, 13 \\ ${ }^{2}$ ООО «Мотив НТ», Москва, 121205, тер. инновац. иентра Сколково, Большой бульвар, 42 с 1.
} эл.nочта:ipdmev@gmail.com

DOI 10.34077/RCSP2019-120

Наступающая эпоха больших данных нуждается в новых вычислительных средствах, способных в режиме реального времени обрабатывать огромные объемы зашумленной информации [1]. Существует множество задач, где разработка чётких алгоритмов с высокой производительностью для традиционных систем является чрезвычайно трудной и часто неосуществимой в разумных временных затратах (например, оптическое распознавание объектов с фотоприёмных приборов, системы контроля производственных процессов и т.д.). Для преодоления этих достаточно жестких ограничений используется машинное обучение искусственных нейронных сетей.

Аппаратная реализация нейронных сетей на центральных процессорах (ЦП) и графических ускорителях (ГП) требует больших энергетических ресурсов, что сильно усложняет применение нейронных сетей в различных областях человеческой деятельности. Современные успехи в области микроэлектроники позволяют разрабатывать и изготавливать интегральные схемы с нейроморфной архитектурой [2], которая старается упрощённо имитировать принципы работы биологических нейронных систем. Такие ИС значительно отличаются от реализации на ЦП и ГП в энергоэффективности и компактности. На данный момент не существует единого решения на счет рационального устройства нейроморфной архитектуры [3,4], поэтому для разработки нейроморфной заказной СБИС существует необходимость в разработке аппаратной инфраструктуры для исследования и пробной эксплуатации импульсных нейронных сетей, построенных с использованием данных СБИС. К такой инфраструктуре относится ряд программных и аппаратных решений, в том числе прототип нейроморфной СБИС на базе программируемых логических матриц в целях апробации основных нейроморфных подходов.

В рамках проекта по разработке нейроморфной СБИС была разработана модульная система, поддерживающая масштабирование размеров моделируемой нейронной сети. Элементарной ячейкой нейронной сети является нейрон, а архитектура предполагает выполнение функций множества нейронов по алгоритму, который реализуется в виде конечного автомата (ядро). Временной шаг работы нейронной сети определяется сигналом тик, за время которого каждое ядро последовательно обрабатывает все свои нейроны. Если потенциал нейрона какого-либо ядра становится достаточно высоким, то он испускает пакет или спайк на заранее указанный адрес дендрита другого нейрона. Объединением ядер в двумерную матрицу с направлениями «Север», «Восток», «Запад» и «Юг»мы получили легко масштабируемую нейронную сеть.

Аппаратная реализация модульной системы состоит из модуля акселератора на базе ПЛИС и объединительной платы. Модуль нейроморфного акселератора на базе ПЛИС представляет собой компактную печатную плату с двумя ПЛИС, которые 131 тысячу нейронов и 67 миллионов синаптических связей. Объединительная плата позволяет устанавливать до 16 модулей нейроморфных акселераторов и предоставляет внешний интерфейс для связи с ПК стандарта USB 3.1. При этом на плате имеются все необходимые скоростные интерфейсы для масштабирования размеров моделируемой нейронной сети за счет объединения плат в корзины и их установки в стойки.

\section{Лuтература}

[1] Hashem et al. The rise of "Big Data" on cloud computing: Review and open research issues. Information Systems, Vol. 47, 2015, pp. 98-115.

[2] B. Benjamin et al. Neurogrid: A Mixed-Analog-Digital Multichip System for Large-Scale Neural Simulations. Proceedings of the IEEE, Vol. 102, No. 5, 2014. pp. 699 - 716.

[3] F. Akopyan et al. TrueNorth: Design and Tool Flow of a $65 \mathrm{~mW} 1$ Million Neuron programmable Neurosynaptic Chip. IEEE Transactions on Computer-Aided Design of Integrated Circuits and Systems, Vol. 34, No. 10, 2015. pp. 1537 - 1557.

[4] M. Davies et al. Loihi: A Neuromorphic Manycore Processor with On-Chip Learning. IEEE Micro, Vol. 38, No. 1, February 2018. pp. $82-99$. 